\title{
Conhecimento dos Enfermeiros Intensivistas de um Hospital Público sobre Despertar
}

\section{Diário: relato de experiência}

\author{
Knowledge of Intensive Care Nurses of a Public Hospital about Daily Awakening: experience
} report

Conocimiento de las enfermeras de cuidados intensivos de un hospital público sobre el Despertar diario: relato de experiencia

\begin{abstract}
Resumo
Objetivo: avaliar o conhecimento do enfermeiro intensivista acerca do despertar diário de pacientes em cuidados críticos. Método: trata-se de um relato de experiência realizado pelas enfermeiras residentes do programa de Atenção a Terapia Intensiva, em um hospital público da cidade de São Paulo, ano de 2021. Resultados: percebeu-se que a prática do despertar diário é de fato pouco utilizada na UTI estudada e, quando iniciada, a atitude geralmente parte da equipe médica, com pouca autonomia dos enfermeiros diante desses casos, evidenciando-se também a falta de protocolo institucional para o embasamento das ações dos enfermeiros frente a essa temática. Conclusão: corroborando com o conceito de que há necessidades educacionais que precisam ser supridas no cenário de cuidados críticos em relação aos profissionais de saúde, especialmente os enfermeiros, finaliza-se propondo duas sugestões ao ambiente destacado, a primeira sendo a promoção da educação permanente em saúde voltada a sedação contínua e o despertar diário dos pacientes críticos, e a segunda, a elaboração e efetivação de um protocolo assistencial nessa vertente, almejando uma prática de saúde baseada em evidências e respaldada institucionalmente.
\end{abstract}

Palavras-chave: Unidades de Terapia Intensiva; Interrupção diária da sedação; Estado de Consciência; Enfermagem de Cuidados Críticos.

\begin{abstract}
Objective: to assess the knowledge of intensive care nurses about the daily awakening of patients in critical care. Method: this is an experience report carried out by nurses residing in the Intensive Care program, in a public hospital in the city of São Paulo, in 2021. Results: it was noticed that the practice of daily awakening is in fact little used in the studied ICU and, when initiated, the attitude usually comes from the medical team, with little autonomy of nurses in these cases, also showing the lack of institutional protocol to support nurses' actions in this area. Conclusion: corroborating the concept that there are educational needs that need to be met in the context of critical care in relation to health professionals, especially nurses, we conclude by proposing two suggestions for the highlighted environment, the first being the promotion of permanent education in health focused on continuous sedation and daily awakening of critical patients, and the second, the development and implementation of a care protocol in this aspect, aiming at an evidence-based and institutionally supported health practice.
\end{abstract}

Keywords: Intensive Care Units; Daily interruption of sedation; State of Consciousness; Critical Care Nursing. 


\begin{abstract}
Resumen
Objetivo: evaluar los conocimientos de los enfermeros de cuidados intensivos sobre el despertar diario de los pacientes en cuidados críticos. Método: se trata de un relato de experiencia realizado por enfermeros residentes en el programa de Cuidados Intensivos, en un hospital público de la ciudad de São Paulo, en el año 2021. Resultados: se notó que la práctica del despertar diario es de hecho poco utilizada en el estudiaron UCI y, cuando se inició, la actitud suele venir del equipo médico, con poca autonomía de las enfermeras en estos casos, lo que también demuestra la falta de protocolo institucional para apoyar la actuación de las enfermeras en esta área. Conclusión: corroborando el concepto de que existen necesidades educativas que deben ser atendidas en el contexto de los cuidados críticos en relación a los profesionales de la salud, especialmente enfermeras, se concluye proponiendo dos sugerencias para el entorno destacado, siendo la primera la promoción de la educación permanente en la salud enfocada en la sedación continua y el despertar diario de los pacientes críticos, y la segunda, el desarrollo e implementación de un protocolo de atención en este aspecto, con miras a una práctica de salud basada en la evidencia y sustentada institucionalmente.

Palabras clave: Unidades de Cuidados Intensivos; Interrupción diaria de la sedación; Estado de consciencia; Enfermería de cuidados intensivos.
\end{abstract}

\title{
1. Introdução
}

A Unidade de Terapia Intensiva, é definida como o local onde se presta assistência em saúde a pacientes críticos, e que para isso dispõem de tecnologia de ponta e atualizada, tendo como finalidade ofertar assistência de qualidade, livre de dados aos pacientes. Entre os diversos recursos encontrados nesses locais, têm-se a ventilação mecânica (VM) e os meios utilizados para manter os pacientes sob esses cuidados confortáveis e sem dor (Montini, Rodrigues, Silveira, Contrin, Werneck, Beccaria., 2020).

Assim, tendo em vista o perfil crítico de saúde desses pacientes, é que nesses locais, são utilizados terapia medicamentosa para obter analgesia e sedação, tendo a finalidade de auxiliar no processo terapêuticos, e mantê-los confortáveis durante a permanência na ventilação mecânica. No entanto, apesar dos efeitos benéficos da sedação e analgesia, essa intervenção precisa ser controlada e avaliada diariamente, pois pode ter impacto negativo direto no despertar diário, ocasionando maior período de permanência na VM (Lima, Silva, Assis, Silva., 2018).

Nesse sentido, objetivando interromper a infusão da sedação, é que se institui na UTI o despertar diário, que é definido como a interrupção diária da sedação, por um período de tempo a cada dia, que tem por finalidade avaliar a indicação de sedação e diminuir o acumulo de fármaco sistêmico, o que permitirá que o paciente fique mais alerta, proporcionando a oportunidade de realizar o desmane precoce da ventilação, contribuindo para minimizar as complicações relacionadas a essa terapêutica (Lima et al., 2018).

Assim, além do despertar diário e da sedação leve, outros métodos tem sido utilizados na prática clínica, objetivando diminuir o período de sedação continua dos pacientes, nesse sentido, é utilizado a sedação intermitente, a qual consiste na administração de doses intercaladas de sedativos, de acordo com as respostas e necessidades clínicas dos pacientes. Vale ressaltar, que apesar de pouco utilizada, essa prática é baseada nas Diretrizes de Prática Clínica de 2013, a qual fomenta a necessidade de controle da dor, delíruim e agitação, por meio da realização de sedação leve, sempre que possível (Lima, Silva, Assis, Silva., 2019).

Estudos apontam a importância da equipe multiprofissional para a realização do despertar diário, sedação leve e a sedação guiada por metas, entretanto, apesar da ação de diversos profissionais, o enfermeiro é considerado como profissional responsável para realizar esse processo nas UTI, agindo esse, de acordo com os protocolos institucionais, e de acordo com as recomendações da equipe multidisciplinar (Barbosa, Beccaria, Bastos, Silva., 2020).

Assim, tendo em vista a importância do enfermeiro para a realização do despertar diário, e sabendo-se das complicações e repercussões clínicas prejudiciais aos pacientes submetidos a sedação excessiva, é que justifica-se a realização desse estudo, o qual irá contribuir com a sociedade contemporânea ao mencionar o déficit de conhecimento dos enfermeiros de uma UTI pública acerca do despertar diário, de modo a promover futuras ações de educação permanente em saúde no 
ambiente de aplicação e indicação de futura elaboração de protocolos assistenciais que visem melhorar o trabalho do enfermeiro no despertar diário.

Nesse viés, diante dos entraves citados, e pretendendo-se alcançar os resultados esperados para essa pesquisa, é que se instituiu os seguintes objetivos para esse estudo: Mencionar o conhecimento do enfermeiro intensivista acerca do despertar diário de pacientes em cuidados críticos em uma UTI de um hospital público municipal; Apontar as falhas de conhecimento de enfermeiros intensivistas quanto a aplicação da dinâmica da interrupção diária da sedação; Descrever as indicações, contraindicações e benefícios do despertar diário em uma unidade de cuidados críticos.

\section{Método}

Trata-se de um relato de experiencia realizado pelas enfermeiras resididentes do programa de Atenção a Terapia Intensiva, em um hospital público da cidade de São Paulo, no ano de 2021. Tal programa é dirigido pela Secretaria Municipal de Saúde do Estado de São Paulo (SMS-SP) e consta em seu regulamento o cumprimento de 60h semanais, alternando entre cenário teórico e prático, com duração extimada de 2 anos.

As enfermeiras residentes, tanto R1 - primeiro ano de residência iniciado em $1^{\circ}$ de março de 2021 , como R2 - segundo ano de residência com início em $1^{\circ}$ de março de 2020 , permanecem em um período de $12 \mathrm{~h}$ no hospital, quatro dias na semana, a saber, segunda, terça, quarta, e as sextas-feiras. Assim, foi optado a descrição do presente artigo por meio do relato de experiência, pois descreve com precisão uma determinada vivência, com o intuito de contribuir para a área de especialização de forma relevante à prática, sendo a descrição feita por um autor ou um grupo de autores com base em sua experiência profissional (Grollmus, Tarrés., 2015).

Considerando a impressão daquele que viveu determinada situação, é produzido de maneira objetiva, sem ser uma narração emotiva ou mera divagação, sendo a impessoalidade e a seriedade características inerentes. A relevância dos relatos de experiência reside na importância das questões levantadas, e os resultados sugerem reflexões com base na experiência relatada.

Trás uma abordagem qualitativa que visa descrever e explicar o fenômeno estudado abordando de diferentes maneiras a partir do entendimento e visão voltada para subjetividade (Flick, 2009). Além disso esse método enfatiza os pesquisadores como instrumento chave do estudo, e o ambiente ser a fonte direta e primordial dos dados, sem precisar utilizar necessariamente uma abordagem estatística (Godoy, 1995).

Tem como objetivo ainda, levantar opiniões e atitudes, proporcionando maior intimidade com o tema relatado por meio do qual os autores/pesquisadores sociais estão preocupados com a prática (Gil, 2002). Este é um estudo em campo prático onde lida-se com situações reais, conforme Pereira et al. (2018) e conta com diversas variáveis e o enredo pode ser analisado (Pereira,

\section{Resultados e Discussão}

Corroborando com as literaturas já mencionadas no decorrer do artigo, percebemos no cotidiano da Residência que a prática do despertar diário é de fato pouco utilizada na UTI estudada e, quando iniciada, a atitude geralmente parte da equipe médica, com pouca autonomia dos enfermeiros diante desses casos, evidenciando-se também a falta de protocolo institucional para o embasamento das ações dos enfermeiros frente á temática. Assim, a maioria dos pacientes intubados que recebem sedoanalgesia ficam nesse estado por um período de tempo significativo, na maioria das vezes com drogas em alta vazão por equipamentos de infusão intravenosa contínua, sendo seu estado neurológico avaliado sem interrupção da sedação. 
Conforme mencionado por Mendes et al., (2019), é sabido que os agentes sedativos têm sua importância e seus benefícios se utilizados da maneira correta, dando preferência a sedação leve, com a realização da interrupção diária da sedação, guiada por uso de protocolos, porém, quando usados de forma inadequada, como a sedação profunda, por longo período, acarretam grandes prejuízos ao paciente levando a piora do seu quadro clínico.

Nesse seguimento, vários danos podem acometer o paciente quando existe uma disparidade em relação á maneira como a sedação está sendo ofertada. Alguns exemplos são a maior permanência na unidade de terapia intensiva, coma, intubação prolongada, alterações neurológicas e a lesão por pressão (Lima et al., 2019)

Todos esses malefícios citados foram observados no cenário de prática, sendo evidenciado que o indivíduo permanece por mais tempo em cuidados críticos, sem previsão precoce de extubação e com risco elevado de desenvolver LPP (Lesão por Pressão), favorecendo um prognóstico clínico ruim e ainda um aumento da taxa de mortalidade e diminuição da sobrevida.

Além dos prejuízos citados acima, devido a infusão de sedoanalgesia em doses altas, Silva et al., (2019), cita a sedação por período prolongado, como um dos fatores de risco para a Pneumonia Associada a Ventilação Mecânica (PAV), patologia essa, que acomete com maior frequência os pacientes internados em UTI, sendo responsável por ocasionar maior período de internação hospitalar, maior período dos pacientes sob ventilação mecânica, piores desfechos clínicos, elevados índices de morbimortalidade e aumento dos custos com a internação.

Nesse sentido, fazendo um link dos fatos literários à prática diária, um estudo realizado em 4 hospitais com participação de 336 pacientes intubados e em uso de sedação, evidenciou a importância do despertar diário associado a tentativa de respiração espontânea como padrão rotineiro a ser instituído nas UTI's, uma vez que, existe uma melhoria evidente no quadro desses indivíduos (Girard, et al., 2008).

Desse modo, existem algumas formas de sedar o paciente que realmente tem indicação para tal conduta, cuja maneira facilita na avaliação de despertar diário, a saber, sedação leve que é uma indicação das Diretrizes de Prática Clínica de 2013, e sedação intermitente, que viabilizam uma melhor avaliação neurológica do paciente e minimiza as complicações atreladas ao uso dessa abordagem terapêutica (Junior, 2015; Lima et al., 2019).

Os sedoanalgésicos mais comumente utilizados na UTI em questão são o midazolam, a fentanila, o propofol e o etomidado, além da dexmedetomidina, corroborando com os descritos na literatura. Apesar de os benzodiazepínicos serem os menos recomendados para a sedação contínua, pois aumentam consideravelmente o tempo em que o indivíduo permanece sedado, sem falar da síndrome de infusão do propofol que provoca rabdomiólise, hipercalemia, acidose metabólica, insuficiência renal e cardíaca, essas são as drogas mais frequentemente utilizadas, inclusive no cenário avaliado (Shehabi, Bellomo, Mehta, Riker, Takala., 2013).

Como observado no cenário de prática hospitalar no âmbito da UTI, a avaliação do despertar diário dos pacientes críticos começou a ganhar mais força e ser realizada com mais frequência no mês de agosto desse ano (2021), o que culminou em maior índice de extubação dos pacientes, sendo esse procedimento realizado de forma segura, pela equipe de fisioterapeutas, culminando em melhores desfechos clínicos dos pacientes, e menor período de internação na UTI.

Conseguintemente, no que concerne ao enfermeiro, segundo o parecer do Conselho Regional de Enfermagem de São Paulo (COREN-SP) número 08 do ano de 2018, este profissional necessita de conhecimentos, habilidades e atitudes que proporcionem uma assistência baseada em evidências científicas com garantias à segurança do paciente, em vista disso, o mesmo parecer dispõe que escalas de avaliação de sedação, agitação e/ou despertar do doente crítico, são de competência privativa dos enfermeiros no que compete as suas funções dentro da equipe de enfermagem, podendo os mesmos serem capazes de tomar condutas mais assertivas e em benefício dos pacientes quando existentes protocolos que os afastem de danos decorrentes de imperícia, negligência ou imprudência. 
Assim, em consonância com a literatura, é preciso ter recursos humanos suficiente e devidamente qualificados para realizar a avaliação do despertar diário. Além do quadro de profissionais estudado ser estreito, existe uma alta rotatividade de pacientes graves e em uso de sedativos, o que dificulta ainda mais a prática do despertar diário pela equipe de enfermagem.

Subsequentemente, um estudo realizado com técnica de randomização e avaliação de 428 participantes, cuja maioria estava sem fármacos sedativos, alocados na unidade de terapia intensiva do Odense University Hospital na Dinamarca, no qual o protocolo padrão é uma prática sem sedação, confirmou que os pacientes sem uso dessa terapêutica permaneceram mais dias sem ventilação mecânica, e menos dias internados no setor de UTI (Strom, Martinussen, Toft., 2010).

Esse contexto mostra o caminhar lento no que diz respeito a interrupção diária da sedação em pacientes críticos, uma vez que, outros países já dispõem de protocolos mais avançados, sem a sedoanalgesia como terapêutica padrão, a não ser nos casos em que os pacientes realmente necessitam, evidenciando que essa prática "sedativa” está associada a maiores prejuízos quando em comparação com os benefícios.

\section{Conclusão}

Diante do exposto, percebe-se que além do despertar diário ser uma prática pouco presente no ambiente relatado, há falhas de conhecimento por parte dos enfermeiros intensivistas quanto as boas práticas de interrupção diária de sedação e avaliação do despertar diário baseado em evidências científicas.

Corroborando para o conceito de que há necessidades educacionais que precisam ser supridas no cenário de cuidados críticos em relação aos profissionais de saúde, é essencial a realização de uma educação permanente em saúde para todos os intensivistas, principalmente os enfermeiros, em relação a temática discutida, objetivando uma melhor adesão dessa prática terapêutica e melhores resultados clínicos dos pacientes, com

redução do tempo de internação e o aumento na taxa de sobrevida, em uma perspectiva de melhoria tanto assistencial, quanto gerencial.

Outro ponto de consideração final nesta pesquisa surge na evidência de déficit não só de capacitação dos profissionais atuantes na UTI, mais também na falta de protocolo institucional que promova a autonomia responsável dos enfermeiros e demais membros da equipe intensivista na interrupção diária das infusões de drogas sedativas e avaliação da evolução dos paciente críticos.

Finaliza-se então, propondo duas sugestões ao ambiente destacado, a primeira sendo a promoção da educação permanente em saúde voltada a sedação contínua e o despertar diário dos pacientes críticos, e a segunda, a elaboração e efetivação de um protocolo assistencial nessa vertente, almejando uma prática de saúde baseada em evidências e respaldada institucionalmente.

\section{Referências}

Aliaga, M. \& Gunderson, B. (2002). Interactive Statistics. Thousand Oaks: Sage.

Barbosa, T. P., Beccaria, L. M., Bastos, A. S. \& Silva, D. C. (2020). Associação entre nível de sedação e mortalidade de pacientes em ventilação mecânica em terapia intensiva. Revista da Escola de Enfermagem da USP.

Barbosa, T. P., Beccaria, L. M., Bastos, A. S., Silva, D. C. (2018). Associação entre sedação e eventos adversos em pacientes de terapia intensiva. Acta Paulista de Enfermagem.

Brasil. Conselho Regional de Enfermagem (2018). Ementa: Aplicação de escalas (EAPM/MEWS, SAS, EDIMBURGO, GLASGOW, DELIRIUM) por profissionais de Enfermagem. COREN-SP 008/2018. https://portal.coren-sp.gov.br/wp-content/uploads/2019/11/08-18.pdf

Chicayban, L. M., Terra, E. L. V. S., Ribela, J. S., \& Barbosa, P. F.(2017). Bundles de prevenção de pneumonia associada a ventilação mecânica: a importância da multidisciplinaridade. Revistas perspectivas online: biológicas \&saúde. Novembro de 2017, Vol. $7^{\circ}$, $\mathrm{n}^{\circ} 25$, p. 25-35. DOI: $10.2524 / 886872520171200$. 
Flick, U. (2009). Desenho da pesquisa qualitativa. Artmed.

Gil, A. C. Como elaborar projetos de pesquisa. (4. ed.) Atlas, 2002.

Girard, T. D., Kress, J. P., Fuchs, B. D., Thomason, J. W., Schweickert, W. D., Pun, B. T., Taichman, D. B., Dunn, J. G., Pohlman, A. S., Kinniry, P. A., Jackson, J. C., Canonico, A. E., Light, R. W., Shintani, A. K., Thompson, J. L., Gordon, S. M., Hall, J. B., Dittus, R. S., Bernard, G. R. \& Ely, E. W. (2008). Efficacy and safety of a paired sedation and ventilator weaning protocol for mechanically ventilated patients in intensive care (Awakening and Breathing Controlled trial): a randomised controlled trial. Lancet. 12;371(9607):126-34. Doi: 10.1016/S0140-6736(08)60105-1. PMID: 18191684.

Godoy, A. S. A. (1995). Pesquisa qualitativa e sua utilização em administração de empresas. Revista de Administração de Empresas. v. 35, n. 4, p.65-71.

Grollmus, S. N. \& Tarrès, J. P. (2015). Relatos metodológicos: difractando experiências narrativas de investigación. Fórum Qualitative Social Research, v. 16, n. 2 .

Junior, A. P. N. (2015). Impacto da sedação intermitente ou interrupção diária da sedação em pacientes sob ventilação mecânica. Faculdade de Medicina da Universidade de São Paulo.

Junior, P. N. J. \& Park, M. (2016). Protocolos de sedação versus interrupção diária da sedação: uma revisão sistemática e metanálise. Revista brasileira de terapia intensiva. 2016;28(4); 444-451.

Lakatos, E. M. \& Marconi, M. A. (2010). Fundamentos da metodologia científica. (7. ed.). Atlas.

Lima, J. T., Silva R. F. A., Assis, A. P. \& Silva, A. (2019). Lista de verificação para gerenciamento do despertar diário de pacientes críticos. Revista brasileira de terapia intensiva.

Lima, J. T., Silva R. F. A., Assis, A. P. \& Silva, A. (2018). Lista de verificação (CHECKLIST) para o gerenciamento do despertar diário de pacientes críticos. Universidade Federal do Estado do Rio de Janeiro.

Mendes, L. C., Mulaski, L. F. S., Figueira, L. A. F., Vieira, L. A. P., Silva, M. M., \& Alves, M. L. C. D. (2019). Sedação de pacientes na unidade de terapia intensiva. Revista Cadernos de medicina. Vol. 02/ N. 03.

Montini, G. R., Rodrigues, M. A. C., Silveira, A. M. S., Contrin, L. M., Werneck, L. A. \& Beccaria, L. M. (2020). Adesão ao bundle para prevenção de pneumonia associada a ventilação mecânica em terapia intensiva. Revista Cuidados de enfermagem.

Pereira A. S., Shitsuka, D. M. \& Shitsuka, F. J. P. R. (2018). Metodologia da pesquisa científica. Ed. UAB/NTE/UFSM.

Silva, G. M., Souza, V. S., Lopes, D., Oliveira, J. L. C., Fernandes L. M., Tonini, N. S., \& Fernandes, C. A. M. (2019). Práticas de prevenção de pneumonia associada á ventilação mecânica em terapia intensiva. Revista enfermagem atual. In derme- 2019, 90-28.

Shehabi, Y., Bellomo, R., Mehta, S., Riker, R. \& Takala, J. (2013). Intensive care sedation: the past, present and the future. Crit Care. 17(3): 322. DOI: $10.1186 / \mathrm{CC} 12679$

Strom, T., Martinussen, T. \& Toft, P. (2010). A protocol of no sedation for critically ill patients receiving mechanical ventilation: a randomised trial. Lancet. 6;375(9713):475-80. DOI: 10.1016/S0140-6736(09)62072-9. EPUB 2010 jan 29. PMID: 20. 